\title{
Effects of biodegradable self-reinforced polyglycolic acid, poly- DL-lactic acid and stainless-steel spiral stents on uroepithelium after Nd: YAG laser irradiation of the canine prostate
}

\author{
A. PÉTAS, P. KÄRKKÄINEN*, M. TALJA†, K. TAARI, M. LAATO $\ddagger$, T. VÄLIMAA§ and P. TÖRMÄLÄ§ \\ Departments of Urology and *Pathology, Helsinki University Central Hospital, †Department of Surgery, Päijät-Häme Central \\ Hospital, Lahti, $\ddagger$ Department of Surgery, Turku University Central Hospital, Turku and §Institute of Biomaterials, Tampere \\ University of Technology, Tampere, Finland
}

Objective To evaluate the safety and tissue effects on canine uroepithelium and prostate of biodegradable self-reinforced polyglycolic acid (SR-PGA) and selfreinforced poly-DL-lactic acid (SR-PLA) spiral stents compared to stainless-steel (SS) spiral stents.

Material and methods Twenty-five dogs (median body weight $12 \mathrm{~kg}$ ) underwent a one-stage midline laparotomy and cystotomy. A side-firing Nd: YAG laser fibre was placed in the prostatic urethra antegradely and laser power applied at a setting of $20 \mathrm{~W}$ for $10 \mathrm{~s}$ in each side lobe, followed by dilatation to $4 \mathrm{~mm}$ diameter and insertion of a spiral stent. Dogs were randomized to receive an SR-PGA, SR-PLA or SS stent and no catheter was placed post-operatively. The dogs were killed after a follow-up of 1, 3 or 6 months and the bladder, prostate and urethra removed. Routine histological sections were prepared from the bladder, prostate, the proximal urethra immediately below the prostate and the distal urethra.

Results Histological examination showed a mild to mod- erate foreign-body reaction and an acute inflammatory reaction after 1 month in the SR-PGA and the SR-PLA groups. The tissue effect was minimal in the SR-PGA group after 6 and 12 months. The SS stent group had more fibrosis, chronic inflammation and oedema at all follow-up assessments. There were erosions of the epithelium with subepithelial oedema in the animals with the SS stent. No dysplasia was detected and some remnants of the suture material were evident in the area of the cystotomy closure. There was one stricture at the apex of prostate in the SR-PGA group at 3 months in one re-operated dog.

Conclusion The histopathological changes in tissues caused by SR-PGA and SR-PLA stents were generally minor and decreased during the follow-up. The biocompatibility of SR-PGA and SR-PLA stents was good when combined with Nd:YAG laser treatment of the prostate.

Keywords Stents, biodegradable, histological effects, prostate therapy, laser

\section{Introduction}

Biodegradable spiral stents made of self-reinforced polyglycolic acid (SR-PGA) and self-reinforced polylactic acid (SR-PLA) are a new generation of devices for temporary stenting of the urethra. These materials have been in clinical use for more than 20 years as suture materials and surgically implanted devices. In an experimental study, SR-PLA showed good biocompatibility in the anterior urethra of the rabbit [1]. The SR-PGA spiral stent has been used in combination with visual laser ablation of the human prostate (VLAP) to ensure postoperative voiding, with promising results [2]. The efficacy and safety of lasers have been studied in dogs and humans [3-7], and VLAP has become an alternative for the treatment of BPH. The therapy is based on photo-

Accepted for publication 7 August 1997 thermal coagulation of prostatic tissue with the Nd:YAG laser and a right-angled side-firing free-beam delivery system. The aim of the present study was to characterize the tissue effects of SR-PGA and SR-PLA stents in combination with Nd:YAG laser irradiation in a canine model, using the stainless-steel (SS) spiral stent as the reference material for comparison.

\section{Materials and methods}

The biodegradable spiral stents were manufactured from PGA or PLA by extrusion and die-drawing [8]; the draw ratio of SR-PGA wire was 4 and for SR-PLA was 7. In the current PLA stent, the wire is a copolymer of $\mathrm{L}^{-}$and D-tereoisomers of lactic acid in a ratio of 96:4. The wire tends to straighten so that the outer diameter increases by about half during incubation for 14 days. The material is biocompatible and degrades in vitro into 
glycolic acid, mainly by hydrolysis [9], the end-products being water and carbon dioxide $[10,11]$. The mechanical properties and the degradation time depend on the degree of polymerization, the internal arrangement of the material components, the site of implantation and the shape of the implant [8]. The degradation time in vivo of SR-PGA is 3-4 weeks and that of SR-PLA 3-6 months.

The configuration of the spiral stents was designed according to the anatomy of the canine urethra. The stents were $4 \mathrm{~mm}$ in diameter, the wire of the SR-PGA and SR-PLA spiral stents was $0.5 \mathrm{~mm}$ (Bioscience Inc, Tampere, Finland) and the wire of the SS was $0.3-0.4 \mathrm{~mm}$ in diameter $\left(\right.$ Cerclage ${ }^{\circledR}$, Aesculap, Germany). SR-PGA and SS spiral stents were sterilized in ethylene oxide and the SR-PLA stents sterilized with gamma irradiation.

\section{Operative procedure}

Twenty-five dogs (median body weight $12 \mathrm{~kg}$, range 10.2-15.0) underwent a one-stage midline laparotomy and cystotomy. A SideFiber $1800^{\circledR}$ (CeramOptec GmbH, Bonn, Germany) laser fibre was placed into the prostatic urethra antegradely without visual control. Nd:YAG laser energy was applied to both side lobes at a power setting of $20 \mathrm{~W}$ for $10 \mathrm{~s} \mathrm{(20} \mathrm{dogs)} \mathrm{and} 40 \mathrm{~W}$ for $20 \mathrm{~s}$ (five dogs) for each coagulation spot. The laser treatment was followed by dilatation of the prostatic urethra to $4 \mathrm{~mm}$ in diameter and insertion of a spiral stent. After treatment, no catheter was placed and the animals were allowed to void and move freely. Enrofloxacin $5 \mathrm{mg} / \mathrm{kg}$ (Baytril ${ }^{\circledR}$, Bayer AG, Leverkusen, Germany) was used for 3 days after treatment as prophylaxis. The dogs were observed to record the beginning of voiding, pain and wound healing. One dog underwent re-operation 2 days after surgery because of urinary retention caused by a blood clot. In that animal, an $8 \mathrm{~F}$ infant-feeding tube was placed retrogradely into the urethra and a new SR-PGA spiral stent placed along the feeding tube antegradely. The end of the feeding tube was fixed to the bladder wall with catgut and detached 5 days later. Before surgery and after death samples were taken to determine haemoglobin content, white blood cell (WBC) count and serum creatinine. A urine sample was taken intra-operatively and at death by aspiration from the urinary bladder. Serum creatinine was measured again after 2 and 4 weeks, and at 3, 6 and 12 months.

The dogs were killed according to the schedule in Table 1. The urinary bladder, prostate and urethra were removed en bloc and cut open longitudinally for macroscopic inspection. Samples were taken for histological examination from the bladder, prostate, proximal urethra immediately below the prostate and the distal urethra. The histological samples were fixed in $10 \%$ phosphate-
Table 1 The number of dogs in the treatment groups and the follow-up times

\begin{tabular}{lcll}
\hline Follow-up (months) & SR-PGA & SR-PLA 96 & Stainless steel \\
\hline 1 & 3 & 2 & 1 \\
3 & 3 & 2 & 2 \\
6 & 3 & 2 & 2 \\
12 & 1 & 2 & 2 \\
Total & 10 & 8 & 7 \\
& & & \\
\hline
\end{tabular}

buffered formalin and embedded in paraffin and sections prepared using the Herovici-van Gieson method. The histological findings were graded and the histopathological effects summarized; the tissue changes in the urinary bladder and the prostate were defined as: erosion (not present 0 , present 1 ), acute inflammation (0-3: not present 0 , mild 1 , moderate 2 , severe 3$)$, chronic inflammation (0-3), necrosis (0-3), dysplasia (0-3), fibrosis (0-3), and foreign-body reaction (0-2). Specimens were also taken for hydroxyproline measurement [12] from the prostate at the site of the cavity on one side. The Mann-Whitney U-test was used to evaluate differences in hydroxyproline values between sample times, with a $P$ value of $<0.05$ considered to indicate statistical significance.

\section{Results}

At the 1-month follow-up, a demarcated lesion, e.g. a cavity or crater in the prostatic side lobes, was noted on gross examination in all dogs in all treatment arms (Table 2). In the SR-PGA group, two dogs treated with a high-energy schedule had side-effects. The first had a

Table 2 A summary of the histological findings for the prostate and urinary bladder. The mean value was calculated for each follow-up assessment using the grading systems described in the methods

\begin{tabular}{lcll}
\hline Stent & $\begin{array}{l}\text { Follow-up } \\
\text { (months) }\end{array}$ & $\begin{array}{l}\text { Prostate } \\
\text { (points/dog) }\end{array}$ & $\begin{array}{l}\text { Bladder } \\
\text { (points/dog) }\end{array}$ \\
\hline PGA & 1 & 4.7 & 1.3 \\
& 3 & 2.3 & 0.3 \\
& 6 & 0.7 & 1.0 \\
PLA 96 & 12 & 1.0 & 1.0 \\
& 1 & 4.5 & 2.5 \\
& 3 & 2.0 & 0.0 \\
Stainless steel & 6 & 2.5 & 1.5 \\
& 12 & 1.0 & 0.5 \\
& 3 & 2.0 & 1.0 \\
& 6 & 3.5 & 3.0 \\
& 12 & 1.0 & 2.5 \\
\hline
\end{tabular}


perforation in the bladder and peritonitis, and histologically showed severe inflammation periurethrally with some calcifications. The epithelium was destroyed in large areas and squamous cell metaplasia, charring and haemosideroblasts were found. Some stent material was left in the lumen. In addition, there was severe inflammation with granulation and calcifications in the proximal urethra. In the other dog, there was macroscopically evident sloughing of tissue, but no stent. Histologically, there was necrosis in large areas with a thin layer of vital prostate, inflamed at the periphery. The third dog in the SR-PGA group at the 1-month follow-up was treated with a lower energy dose. Gross examination revealed only small cavities in the prostatic side lobes, and a healthy-looking bladder and urethral mucosa; stent fragments were absent. Histology revealed chronic inflammation and squamous cell metaplasia in one area. In both dogs in the SR-PLA group at 1 month, the intact stent had migrated distally. Histologically, chronic inflammation and many calcifications were found in both dogs, with some foreignbody reactions in one. Only one dog remained in the SS stent group at 1 month, and this animal had a macroscopically thick bladder wall, with the stent still in place. Histologically, there was moderate non-specific inflammation, thrombosis, minor oedema and haemosideroblasts.

At the 3-month follow-up, three dogs in the SR-PGA group had no evidence of stents. In the re-operated dog, a stricture was evident at the apex of the prostate. Histologically, there was a foreign-body reaction and many calcifications (Fig. 1a). The two dogs in the SR-PLA 96 group at the 3-month follow-up had many small curved and circular stent fragments in the bladder; the changes in histology were minimal. Two dogs in the SS stent group had the stent macroscopically in the correct position with no stones or encrustation. There was moderate inflammation, and squamous cell metaplasia.

At the 6-month follow-up, gross examination and histology of the three dogs in the SR-PGA group showed minimal changes with only small cavities in the prostatic lobes. Two dogs in the SR-PLA group also had minor changes; the stent had disappeared and there were no stones. One had prostatitis with epithelial dysplasia, but the other had no tissue changes (Fig. 1b). Two dogs in the SS stent group had a reddish bladder wall with the stent correctly placed, and no stones or encrustation. One had a cyst $1.5 \mathrm{~cm}$ in diameter in the left lobe of the prostate. Histology showed moderate and minor chronic inflammation and haemosiderophages.

At the 1-year follow-up, gross examination of the one dog in the SR-PGA group, the bladder was normal and the prostate had large cavities in the lobes; there
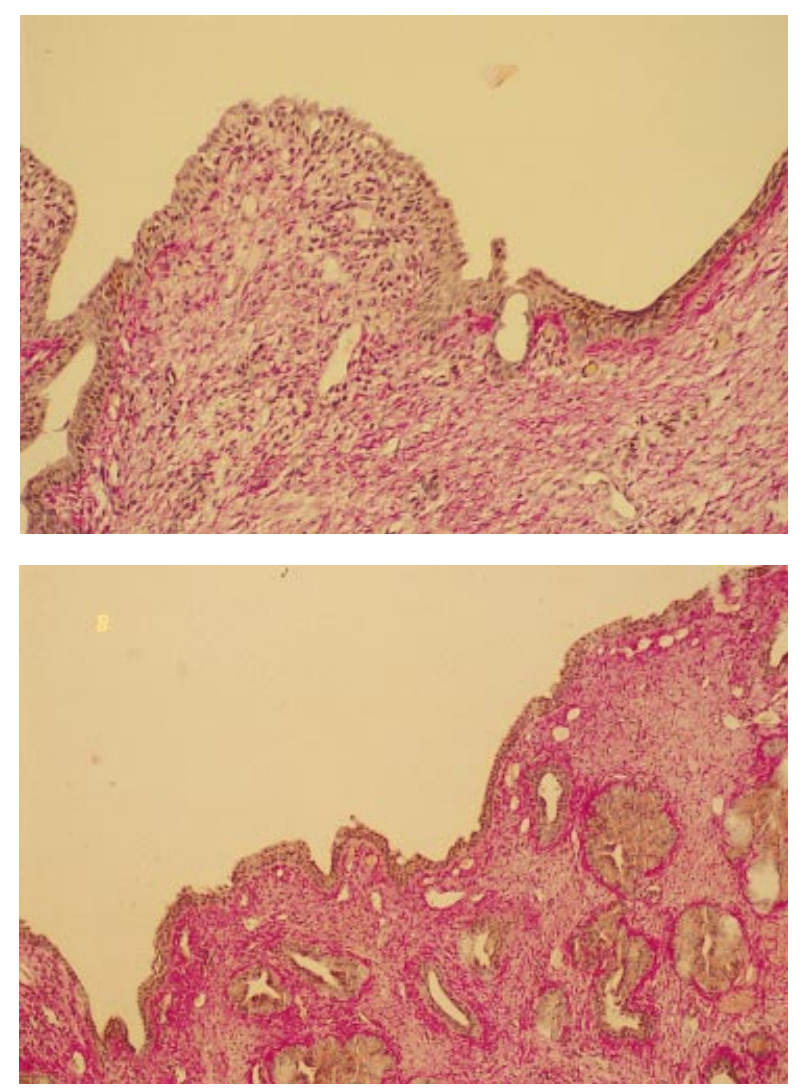

Fig. 1. Prostate obtained, a, 3 months after VLAP and insertion of an SR-PGA stent, showing mild subepithelial inflammation of the prostatic urethra and occasional squamous cells. Herovici-van Gieson. $\times 200$; and, b, 6 months after VLAP and insertion of a SR-PLA stent, showing the prostatic necrotic tissue sloughed off and the lumen covered with normal epithelium. Herovici-van Gieson. $\times 100$.

was very mild chronic inflammation. The two dogs in the SR-PLA group had similar gross findings and no histological changes. In the SS stent group, two dogs had the stent in the correct position and the urethra was reddish; one had three stones $5 \mathrm{~mm}$ in diameter in the bladder, and histologically one had chronic inflammation, subepithelial proliferation of blood vessels and epithelial hyperplasia. The other had erosions, subepithelial inflammation, partial desquamation and atypia of the epithelium with mitotic activity, and calcifications.

The histopathological effects on the prostate and urinary bladder are summarized in Table 2. The histological changes were minor in the proximal urethra. Severe inflammation, granulations and calcifications were found in the SR-PGA group treated with a highenergy dose at the 1-month follow-up. In addition, the two dogs in the SS stent group that were also treated with a high-energy dose had some squamous cell metaplasia and minor inflammation at the 1-year 
follow-up. There were no histological changes in any dog in the distal urethra, which was outside the contact area of the stent.

The stent material was not embedded or incorporated into the tissues in any of the histological samples. Some remnants of suture material were evident at the area of cystotomy closure sites, in a few cases for up to 3 months. Urine analysis revealed no infection; the mean (range) creatinine level was $71(56-84) \mu \mathrm{mol} / \mathrm{L}$ before surgery, $66(54-79)$ at 2 weeks, $72(67-81)$ at 1 month and $73(62-86) \mu \mathrm{mol} / \mathrm{L}$ at death, with no significant differences. The hydroxyproline analysis showed no significant differences between the groups.

\section{Discussion}

Both stents and lasers have previously been described for the treatment of $\mathrm{BPH}$ in clinical practice. Although PGA and PLA have been used in daily surgical practice for many years, the combination of Nd:YAG laser irradiation with a biodegradable self-reinforced spiral stent in the urethra is new and requires analysis of any interaction.

The present study suggests that adverse effects on the prostate and bladder epithelium are minimal; at 1-3 months, the macroscopic and histological findings for SR-PGA, SR-PLA and SS stents were similar in the transitional cell epithelium. Later, the amount of fibrosis, oedema and inflammation increased in the SS stent group; erosions of the epithelium with subepithelial oedema was seen only in this group. This is logical, as after the biodegradable material is lost there can be no mechanical irritation. SR-PGA and SR-PLA are weak acids, but their degradation is so slow that changes in $\mathrm{pH}$ are insignificant. Although studies in humans have shown no evidence of stones on SR-PGA stents, one case did occur in the present study, with another dog in the SS stent group having three large stones. There was no encrustation of the stents.

There is an effect of Nd:YAG laser irradiation on prostatic tissue and some of the tissue change may be cause by laser treatment. Reports suggest that laser effects on the canine prostate are cellular and interstitial oedema, vascular thrombosis and increased interstitial haemorrhage, occuring over 3-7 days. Necrotic areas then develop gradually, followed by cavitation. Proliferation of epithelium with squamous cell metaplasia is a distinct feature, which is not considered a pre-malignant condition. Healing is achieved within a few weeks after laser treatment [13]; the initial re-epithelialization of the resulting cavity occurs at 3 weeks, with nearly complete epithelialization 7 weeks after laser treatment [4]. The present series included four cases of squamous cell metaplasia and the results were comparable with other published histopathological findings at 1 and 3 months.

Video laparoscopy can provide access to the canine bladder and prostatic urethra to allow VLAP without either open laparotomy or urethrostomy. Van-Lue et al. [15] were successful with a video-assisted technique for all eight of their dogs. In the present open method, access to the prostatic urethra was also antegrade. However, early in the series, the lack of visual control of the laser beam caused complications.

The canine prostate is composed mainly of glandular elements with little stroma, which causes the effect of laser prostate therapy to differ from that in the human prostate; coagulation in the latter may not be homogeneous [16]. Costello et al. reported histopathological changes after Nd:YAG coagulation in patients who subsequently underwent prostatic resection or open prostatectomy. Initially the changes were akin to those after a thermal burn; there was an immediate loss of cellular integrity followed by an inflammatory cell infiltration and subsequent tissue necrosis. Evidence of necrotic tissue in the resection specimen 10 months after laser application, together with the presence of vascular thrombosis in the periphery of viable gland, suggests that continuing ischaemia may contribute to the pathological changes [6].

Hydroxyproline is almost always found in collagens and thus widely used as an indicator of collagen synthesis. Earlier studies revealed that serious fibrotic obliteration is possible and depends upon the material of the stent $[17,18]$. However, there were no significant differences in the mean amount of hydroxyproline in the three groups in the present study, indicating that no risk of fibrosis of the urethra is to be expected, at least up to one year after treatment.

Late adverse effects, e.g. perforation, fibrosis and retention caused by stent fragments, did not occur in dogs with SR-PGA or SR-PLA stents; the one case of apical stricture was assumed to be caused by the re-operation and use of a catheter, which remained in situ for an additional 5 days.

These results provide an insight into the effects of implanted biodegradable materials on irradiated prostatic tissue and on intact uroepithelium. That histology revealed no signs of malignant transformation supports earlier findings on these materials, which have shown good biocompatibility [19]. Although differences between the canine and human prostate are evident, we assume that the results of these experimental methods in the dog would be similar to those in humans. As the histopathological changes during the follow-up were minimal at the 1-year follow-up in the SR-stented groups we conclude that the biocompatibility of SR-PGA and SR-PLA stents is good when combined with Nd:YAG laser treatment. 


\section{Acknowledgements}

This study was supported by grants from Technology Development Centre, Helsinki, Finland; The Finnish Urological Association; and Pharmacia, Inc. We thank the personnel of the National Animal Center, Kuopio, Finland, for excellent care of the canines throughout the study. We thank Leena Reinikainen MD for help in the calculation for the statistical assays.

\section{References}

1 Kemppainen E, Talja M, Riihelä M, Pohjonen T, Törmälä P, Alfthan O. A bioresorbable urethral stent. Urol Res 1993; 21: $235-8$

2 Talja M, Tammela T, Pétas A et al. Biodegradable selfreinforced polyglycolic acid spiral stent in prevention of postoperative urinary retention after visual laser ablation of the prostate-laser prostatectomy. J Urol 1995; 154: 2089-92

3 Kandel BK, Harrison LH, McCullough DL, Woodruff RD, Dyer RB. Transurethral laser prostatectomy in the canine model. Lasers Surg Med 1992; 12: 33-42

4 Kabalin JN, Gill HS. Dosimetry studies utilizing the Urolase right angle firing neodymium:YAG laser fiber. Lasers Surg Med 1994; 14: 145-54

5 Perlmutter AP, Muschter R. The optimization of laser prostatectomy. Part I: Free beam side fire coagulation. Urology 1994; 44: 847-55

6 Costello AJ, Bolton DM, Ellis D, Crowe H. Histopathological changes in human prostatic adenoma following neodymium:YAG laser ablation therapy. J Urol 1994; 152: 1526-9

7 Pow-Sang M, Orihuela E, Motamedi M et al. Thermocoagulation effect of diode laser radiation in the human prostate: acute and chronic study. Urology 1995; 45: 790-4

8 Törmälä P. Biodegradable self-reinforced composite materials; manufacturing structure and mechanical properties. Clin Mater 1992; 10: 29-34

9 Chu CC. Hydrolytic degradation of polyglycolic acid: tensile strength and crystallinity study. J Appl Polym Sc 1981; 26: $1727-34$
10 Williams DF, Mort E. Enzyme-accelerated hydrolysis of polyglycolic acid. J Bioeng 1977; 1: 231-8

11 Chu CC, Williams DF. The effect of gamma irradiation on the enzymatic degradation of polyglycolic acid absorbable sutures. J Biomed Mater Res 1983; 17: 1029-40

12 Woessner JF Jr. The determination of hydroxyproline in tissue and protein samples containing small proportions of this amino acid. Arch Biochem 1961; 93: 440-7

13 Pow-sang M, Orihuela E, Motamedi M et al. Healing response of the canine prostate to Nd: YAG laser radiation. Prostate 1996; 28: 287-94

14 Cromeens DM, Price RE, Johnson DE. Pathologic changes following transurethral canine prostatectomy with a cylindrically diffusing fiber. Lasers Surg Med 1994; 14: 306-13

15 Van Lue SJ, Cowles RS III, Rawlings CA. Video-assisted percutaneous cystoscopy of the bladder and prostatic urethra in the dog: new approach for visual laser ablation of the prostate. J Endourol 1995; 9: 503-7

16 Orihuela E, Motamedi M, Pow-Sang M LaHaye M, Cowan D. Warren M. Histopathological evaluation of laser thermocoagulation in the human prostate: optimiziation of laser irradiation for benign prostatic hyperplasia. J Urol 1995; 153: 1531-6

17 Baert L, Verhamme L, van Poppel H, Vandeursen H, Baert J. Long-term consequences of urethral stents. J Urol 1993; 150: 853-5

18 Verhamme L, van Poppel H, van de Voorde W, Baert L. Total fibrotic obliteration of urethral stent. Br J Urol 1993; 72: 389-90

19 Vainionpää S. Biodegradation of polyglycolic acid in bone tissue: an experimental study on rabbits. Arch Orthop Trauma Surg 1986; 104: 333-8

\section{Authors}

A. Pétas, MD, Consultant in Urology.

P. Kärkkäinen, MD, PhD, Consultant in Pathology.

M. Talja, MD, PhD, Senior Lecturer.

K. Taari, MD, PhD, Consultant in Urology.

M. Laato, MD, PhD, Consultant in Urology.

T. Välimaa, GE(Eng), Project Manager.

P. Törmälä, Professor

Correspondence: Dr A. Pétas, Department of Urology, Helsinki University Central Hospital, Haartmaninkatu 4, FIN-00290 Helsinki, Finland. 